\title{
Detection and genotyping of human adenovirus and sapovirus in children with acute gastroenteritis in Belém, Pará, between 1990 and 1992: first detection of GI.7 and GV.2 sapoviruses in Brazil
}

\author{
Larissa Cristina Prado das Neves Costa ${ }^{[1]}$, Jones Anderson Monteiro Siqueira ${ }^{[2]}$, \\ Thayara Morais Portal ${ }^{[3]}$, Edivaldo Costa Sousa Júnior ${ }^{[3]}$, Alexandre da Costa Linhares ${ }^{[2],}$ \\ Yvone Benchimol Gabbay ${ }^{[2]}$ and Hugo Reis Resque ${ }^{[2]}$
}

\begin{abstract}
[1]. Programa de Pós-Graduação em Biologia Parasitária na Amazônia, Universidade do Estado do Pará, Belém, PA, Brasil. [2]. Seção de Virologia, Instituto Evandro Chagas, Secretaria de Vigilância em Saúde, Ministério da Saúde, Ananindeua, PA, Brasil. [3]. Programa de Pós-Graduação em Virologia, Instituto Evandro Chagas, Secretaria de Vigilância em Saúde, Ministério da Saúde, Ananindeua, PA, Brasil.
\end{abstract}

\begin{abstract}
Introduction: Acute gastroenteritis (AGE) is one of the most common causes of morbidity and mortality, especially among children from developing countries. Human adenovirus (HAdV) and sapovirus (SaV) are among the agents that cause AGE. The present study aimed to detect and genotype HAdV and $\mathrm{SaV}$ in 172 fecal samples from children with AGE, collected during a surveillance study carried out in a low-income community in Belém, Pará, between 1990 and 1992. Methods: HAdV was detected by nested PCR, using primers Hex1deg/Hex2deg and NeHex3deg/NeHex4deg. SaV was assayed by reverse transcription PCR (RT-PCR), nested PCR, and quantitative PCR. The nucleotide sequence was determined by direct cycle sequencing. Results: Overall, $43 \%$ (74/172) of samples were positive for HAdV, of which $70.3 \%(52 / 74)$ were sequenced and classified as belonging to five different species, mostly A and F. For SaV, positivity was 5.2\% (9/172) and genotypes GI.1, GI.7, GII.1, and GV.2 were detected. Conclusions: The present results reinforce the need for further studies to obtain epidemiological data about the circulation of these viruses in Brazil, especially in the Amazon Region, where data from the early 1990's are scarce. Furthermore, the study describes for the first time the detection of SaV genotypes GI.7 and GV.2 in Brazil, showing that these types circulated in the region more than 25 years ago.
\end{abstract}

Keywords: Gastroenteritis. Children. Adenovirus. Sapovirus.

\section{INTRODUCTION}

Acute gastroenteritis (AGE) remains a serious global health problem, especially among children in developing countries. It is estimated that more than 700,000 deaths occur annually in children below the age of five due to episodes of acute infectious diarrhea, with approximately $70 \%$ of these cases being caused by enteric viruses. Among the major causative viral agents of AGE are rotaviruses (RV), caliciviruses [norovirus (NoV) and sapovirus $(\mathrm{SaV})]$, human astroviruses (HAstV), and enteric adenovirus (AdE) ${ }^{1}$.

There are currently 70 human adenovirus (HAdV) types arranged in seven species (A-G) ${ }^{2}$. HAdV belongs to the family Adenoviridae, genus Mastadenovirus, and presents a diameter

Corresponding author: Dr. Hugo Reis Resque.

e-mail: hugoresque@iec.pa.gov.br

Received 9 June 2017

Accepted 19 September 2017 of 70-90nm. HAdV displays an icosahedral symmetry, is nonenveloped, and possesses a double-stranded deoxyribonucleic acid (DNA) genome. AdE infection varies according to the species. Types 40 and 41 are related to the increased prevalence of AGE sporadic cases and outbreaks in children. Other species, such as A (serotypes 12, 18, and 31) and G (serotype 52) are also associated with gastroenteritis episodes ${ }^{3}$.

Sapovirus is an etiological agent of both outbreaks and sporadic cases of human AGE detected in all ages. It belongs to the Caliciviridae family, has an icosahedral symmetry, is a non-enveloped virus, has a diameter of $27-40 \mathrm{~nm}$, and possesses a single-stranded ribonucleic acid (RNA) genome. It is classified into five genogroups (GI-GV), of which GI, GII, GIV, and GV are associated with AGE in humans ${ }^{3}$.

Epidemiological data have shown that both viruses are circulating around the world, with prevalence rates of 16.2$19.3 \%$ for HAdV detected by immunochromatography ${ }^{4,5}$ and $0.8-5 \%$ for $\mathrm{SaV}^{6,7}$ detected by reverse transcription polymerase chain reaction (RT-PCR). In Brazil, some investigations have 
reported $\mathrm{HAdV}$ and $\mathrm{SaV}$ infections among hospitalized children in the Northern Region, with prevalence rates of $2-10 \%$ and 2.5-5.9\%, respectively, based on enzyme immunoassay (EIA), PCR, and RT-PCR methodologies ${ }^{8-11}$.

With the advent of molecular techniques, it is now possible to detect and genotype enteric viruses, and thus improve our knowledge of their molecular epidemiological profiles. This is particularly important in the Northern Region of Brazil, where data on the occurrence of such viruses are scarce.

The present study aimed to investigate the occurrence of $\mathrm{HAdV}$ and $\mathrm{SaV}$ infections at the community level, utilizing a collection of fecal samples from children, who were followed up for acute gastroenteritis during 1990-1992 in Belém, Northern Brazil.

\section{METHODS}

\section{Patients and specimens}

Fecal specimens were collected from 540 children, who were enrolled in a 2-year, prospective, double-blind, placebocontrolled, randomized trial, conducted in the community of Belém, Brazil, from April 1990 to July 1992. In that study, the infants received three doses of placebo or tetravalent rhesushuman reassortant rotavirus vaccine (RRV-TV) and were followed up from birth to two years of age $\mathrm{e}^{12,13}$.

Of the 3,075 samples collected throughout the study period, 1,990 were from diarrheic children, of which $4.6 \%(91 / 1,990)$ and $5.4 \%(34 / 624)$ were positive for rotavirus and astrovirus, respectively ${ }^{13,14}$. Of the remaining 1,865 rotavirus- andastrovirus-negative samples, we randomly selected a subset of 172 samples, which represented approximately $10 \%$ of the overall samples collected during each month. Those samples were further tested for the presence of $\mathrm{HAdV}$ and $\mathrm{SaV}$ with a sampling error of $5 \%$ and a confidence interval of $95 \%$. All fecal specimens were kept frozen during these years, at a temperature of $-20^{\circ} \mathrm{C}$, stored in sterile containers, and manipulated at times.

\section{Ethical considerations}

Research involving HAdV and $\mathrm{SaV}$ was approved by the Human Research Ethics Committee of the Evandro Chagas Institute (Approval Number 1,053,357 dated April 30 $0^{\text {th }}$, 2015).

\section{Fecal suspensions}

Fecal suspensions were diluted in $0.01 \mathrm{M}$ Tris/ $/ \mathrm{HCl} / \mathrm{Ca}^{2+}$, $\mathrm{pH} 7.2$, buffer, to a final concentration of $10 \%(\mathrm{w} / \mathrm{v})$.

\section{Viral RNA extraction}

Samples were subjected to total nucleic acid extraction using a commercial PureLink ${ }^{\circledR}$ Viral RNA/DNA Mini Kit (Invitrogen, Carlsbad, CA, USA), according to the manufacturer's instructions.

\section{Molecular detection}

Adenovirus: nested PCR was performed for HAdV detection and genotyping, using primer pairs Hex $1 \mathrm{deg}$ (5'-GCC SCA RTG GKC WTA CAT GCA CAT C-3'), Hex2deg (5'-CAG CAC SCC ICG RAT GTC AAA-3'), and NeHex3deg (5'-GCC CGY GCM
ACI GAI ACS TAC TTC-3'), NeHex4deg (5'-CCY ACR GCC AGI GTR WAI CGM RCY TTG TA-3') in the first and second rounds, respectively. These primers amplify a region of 301 base pairs (bp) by PCR and $171 \mathrm{bp}$ by nested PCR, respectively ${ }^{15}$.

Sapovirus: reverse transcription, using a random hexamer primer, was perfomed to yield the complementary deoxyribonucleic acid (cDNA). Subsequently, samples were subjected to two different PCRs, with primers p289 (5'-TGA CAA TGT AAT CAT CAC CAT A-3') and p290 (5'-GAT TAC TCC AAG TGG GAC TCC AC-3' ${ }^{16}$ to produce a fragment of $331 \mathrm{bp}$, and SVF13 (5'-GAY YWG GCY CTC GCY ACC TAC-3'), SV-F14 (5'-GAA CAA GCT GTG GCA TGC TAC-3'), SV-R13 (5'-GGT GAN AYN CCA TTK TCC AT-3'), and SV-R14 (5'-GGT GAG MMY CCA TTC TCC AT-3') to generate a fragment of $800 \mathrm{bp}$. Additionally, nested PCR was performed with primers SV-F22 (5'-SMW AWT AGT GTT TGA RAT G-3') and SV-R2 (5'GWG GGR TCA ACM CCW GGT GG-3'), with an amplicon of $420 \mathrm{bp}^{17}$. Finally, quantitative polymerase chain reaction (qPCR) was performed with the Super Script III Platinum One-Step Quantitative RT-PCR System kit (Qiagen, Hilden, Germany) for the detection of all $\mathrm{SaV}$ genogroups within the same reaction ${ }^{18}$.

\section{Nucleotide sequencing}

Samples undergoing nucleotide sequencing were purified with the QIAquick ${ }^{\circledR}$ PCR Purification and QIAquick ${ }^{\circledR}$ Gel Extraction (Qiagen) commercial kits according to the manufacturer's instructions.

Sequencing was performed on all samples whose PCR products were of sufficient quantity and quality for further analyses. The primers used were the same as those used for the PCR and nested PCR. We used the Big Dye Terminator Kit Cycle Sequencing Ready Reaction Kit (v. 7.0) (Applied Biosystems, Foster City, CA, USA) and sequences were compared using the BLAST tool with cognate sequences available in the GenBank database.

\section{Sequence assembly and editing}

The nucleotide sequence was determined by direct cycle sequencing and the chromatograms were analyzed using the Geneious 9.1.8 software and compared with other sequences deposited in the GenBank database ${ }^{19}$.

\section{Genotyping}

Genotyping was performed using the IQ-TREE program (v.1.3.2) to choose the most appropriate evolutionary model and maximum likelihood analysis. A non-parametric test with a bootstrap of 1000 replicates was applied. Figtree (v.1.4.2) software was used to edit the generated phylogenetic tree ${ }^{20}$. The sequences obtained in this study were deposited in the GenBank database under accession numbers KY883386-KY883441.

\section{Statistical analysis}

Statistical analysis was performed using BioEstat 5.0 software $^{21}$. The G test with Yates correction for independent samples was used to set up comparisons of positive cases to the age groups studied; a $\mathrm{p}$ value $<0.05$ was considered to indicate statistical significance. 


\section{RESULTS}

Human adenovirus was detected in $43 \%$ (74/172) of samples, of which $70.3 \%(52 / 74)$ were sequenced, resulting in the characterization of five different species (Table 1). Nine (5.2\%) out of the 172 tested samples were found to be SaV-positive by qPCR. Of these, 55.5\% (5/9) were positive by nested PCR and PCR. Of all the SaV-positive samples, 44.4\% (4/9) were sequenced.

Molecular analyses showed that HAdV species A and F were the most prevalent, accounting for $28.8 \%$ and $26.9 \%$ of isolates, respectively. Species B, C, and D could also be identified albeit at lower positivity rates: $7.7 \%, 21.1 \%$, and $15.4 \%$, respectively (Figure 1 and Table 1).

Four SaV-positive samples showed good-quality products and were further analyzed, yielding genotypes GI.1, GI.7, GII.1, and GV.2 (Figure 2).

\section{TABLE 1}

Adenovirus species and types detected in 52 positive fecal samples from children with gastroenteritis in Belém, Pará, Northern Brazil, between 1990 and 1992.

\begin{tabular}{lcc}
\hline Species (\%) & Types & Samples \\
\cline { 2 - 3 } & & $\mathbf{n}(\mathbf{\%})$ \\
\hline A (28.8) & 12 & $9(17.3)$ \\
& 18 & $1(1.9)$ \\
B (7.7) & 31 & $5(9.6)$ \\
C (21.2) & 3 & $4(7.7)$ \\
& 1 & $8(15.4)$ \\
D (15.4) & 2 & $2(3.8)$ \\
& 6 & $1(1.9)$ \\
F (26.9) & 19 & $7(13.5)$ \\
& 51 & $1(1.9)$ \\
Total (100.0) & 40 & $6(11.5)$ \\
\hline
\end{tabular}

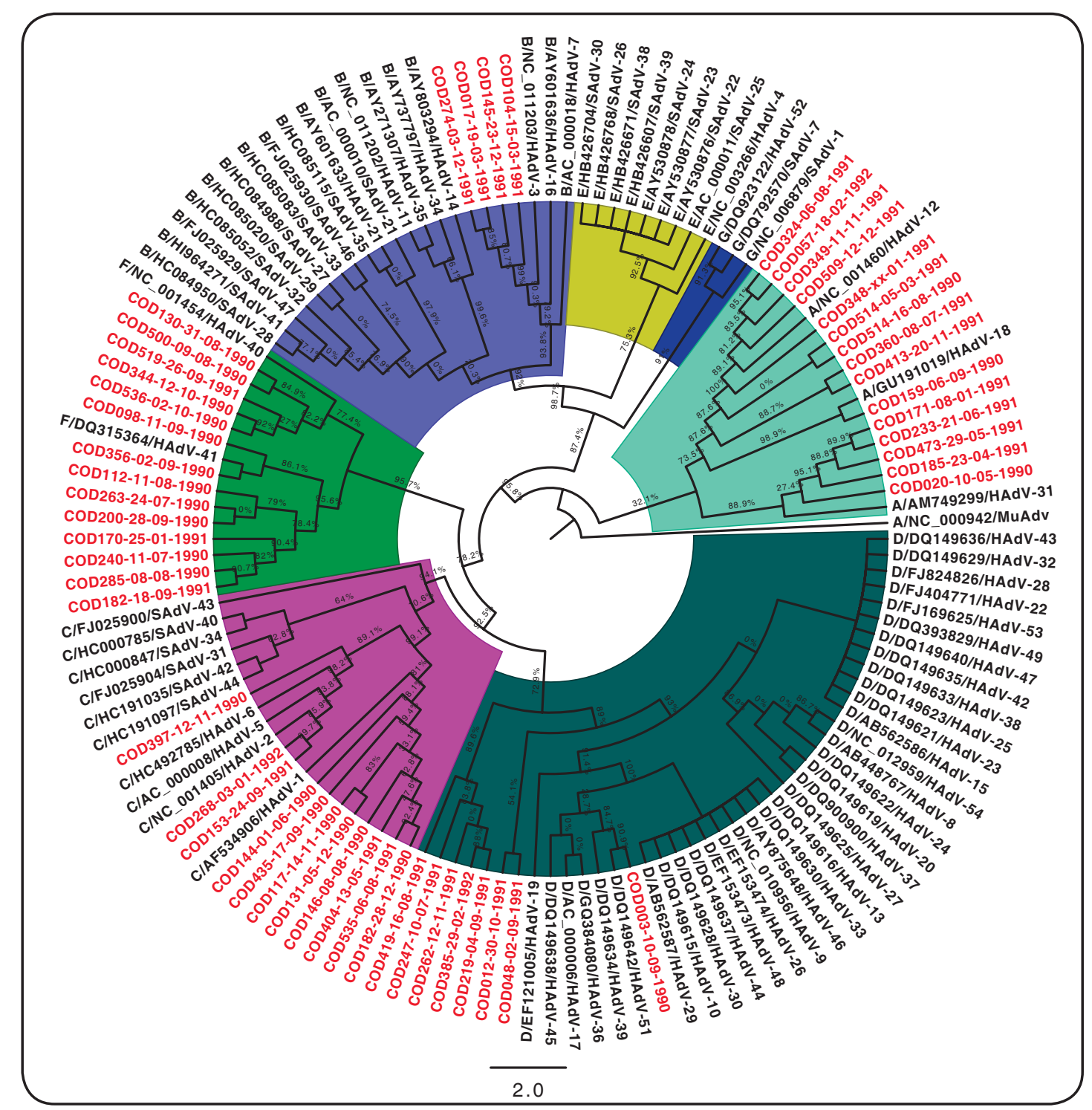

FIGURE 1 - Phylogenetic analysis based on the hexon gene of human adenoviruses detected in 52 samples from children with gastroenteritis in Belém, Brazil, between 1990 and 1992. HAdV: human adenovirus. 

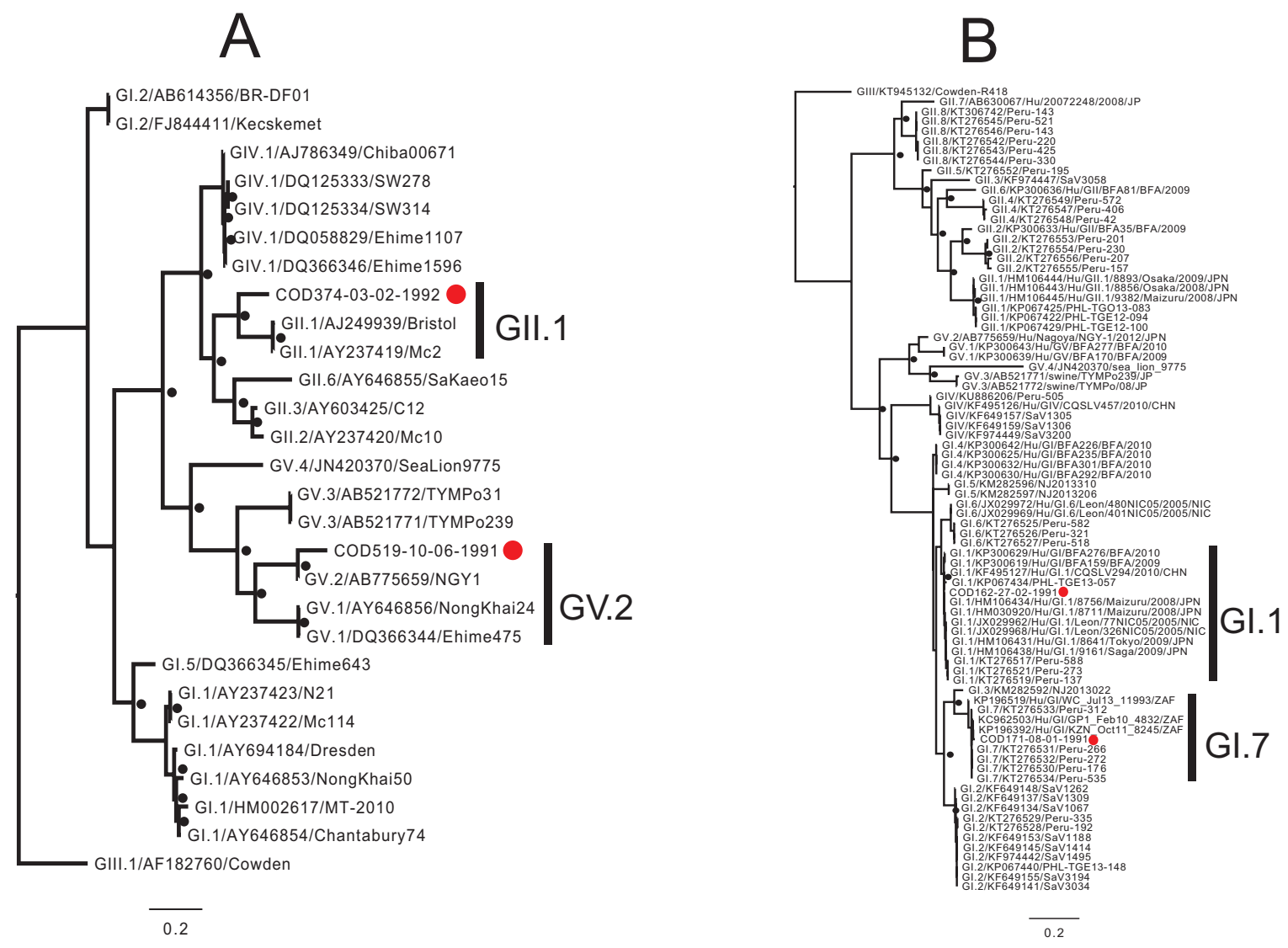

FIGURE 2 - Phylogenetic trees based on the partial sequencing of the (A) polymerase and (B) capsid regions of sapovirus (331bp and 410bp, respectively) using the maximum likelihood method with General Time Reversible (GTR) evolution model analysis of 1,000 bootstrap replicates.

The stratification by age groups showed a slight increase in the number of HAdV-positive cases among children aged 18-24 months, but without being statistically significant according to the chi-square test $[\mathrm{G}=1.33 ; \mathrm{p}=0.24$ (Table 2)]. With regard to $\mathrm{SaVs}$, a higher frequency was demonstrated in the 18-24 months age group, which was statistically significant compared to the remaining age groups $(\mathrm{G}=6.21 ; \mathrm{p}=0.01)$.

With regard to the HAdV monthly distribution, a typical seasonal pattern was not apparent, even though a $100 \%$ positivity rate was achieved in the months of September and
December 1991 (Figure 3). In contrast, SaV circulated mostly during the months corresponding to the rainy season in the Amazon: in 1991, January $(\mathrm{n}=2)$, February $(\mathrm{n}=2)$, and June $(n=1)$; and in 1992, January $(n=1)$, February $(n=2)$, and March $(n=1)$. There were no SaV-positive cases in 1990 .

\section{DIsCussION}

Childhood gastroenteritis remains a major cause of morbidity and mortality worldwide, particularly in individuals younger than five years. In the present study, we observed

TABLE 2

Prevalence rates by age of adenovirus- and sapovirus-positive cases detected in children with acute gastroenteritis between 1990 and 1992 .

\begin{tabular}{|c|c|c|c|c|}
\hline $\begin{array}{l}\text { Age group } \\
\text { (months) }\end{array}$ & $\begin{array}{c}\text { Adenovirus } \\
\text { positive/total (\%) }\end{array}$ & $p$ value & $\begin{array}{c}\text { Sapovirus } \\
\text { positive/total (\%) }\end{array}$ & $p$ value \\
\hline $1-6$ & $28 / 64(43.7)$ & 0.9911 & $0 / 64(0.0)$ & 1.0000 \\
\hline $12-18$ & $23 / 52(44.2)$ & 1.0000 & $3 / 52(5.8)$ & 0.8682 \\
\hline $18-24$ & $12 / 21(57.1)$ & 1.0000 & $4 / 21(19.0)$ & 0.0346 \\
\hline
\end{tabular}




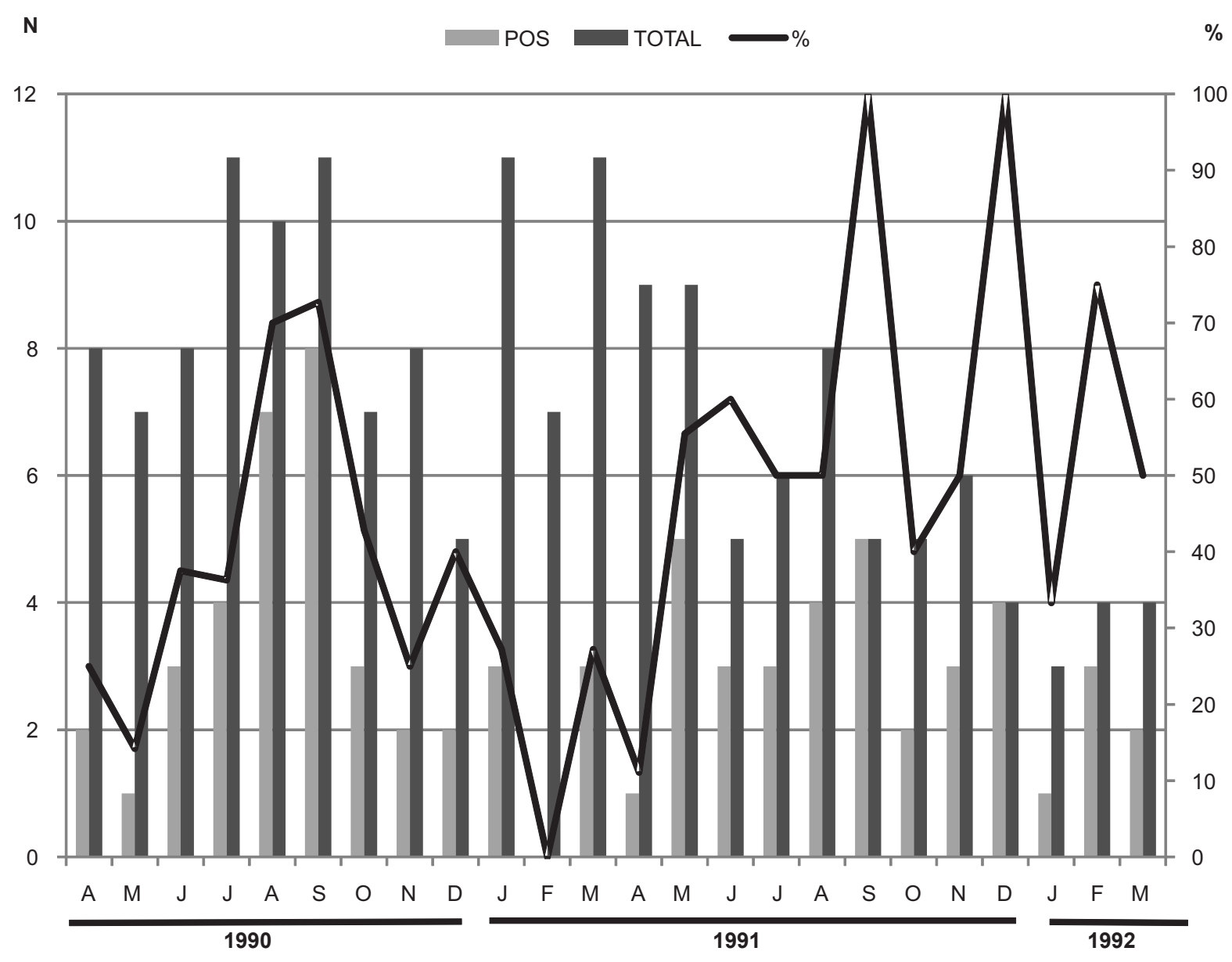

FIGURE 3 - Monthly distribution of adenovirus-positive cases observed in children with gastroenteritis, collected from April 1990 to March 1992. POS: positive; \%: percentage.

a higher prevalence of HAdV infection (43\%) in children followed up in the community than in hospitalized children, as reported in Sudan (16.2\%), Tanzania (3.5\%), and Albania $(23.2 \%)^{5,22,23}$. The higher values could be attributed to a number of factors. For one, 20 years ago when our samples were collected, the overall situation, such as different health awareness, population awareness, sanitary norms, access to health/vaccination programs, was different from that encountered in more recent studies. Nevertheless, a subset of the children from the present study were vaccinated with a rotavirus vaccine (RRV-TV) that was $75 \%$ effective against pure rotavirus infection ${ }^{24}$. Similar results were observed with the current rotavirus vaccine (strain RIX4414, GlaxoSmithKline-GSK, Belgium), reflecting the situation in other countries. Second, in the years 1990-1992, there was no access to methodologies as sensitive as those used today for detecting viruses. Third, the primers used in this study can detect all types of HAdV (enteric and non-enteric) that have the capacity to replicate in the gut and are excreted in the feces. Fourth, in this study, a nested PCR technique was used, which has greater sensitivity than the commonly used EIA. In contrast, for $\mathrm{SaV}$, the proportion in our study $(5.2 \%)$ was lower than that reported previously in Brazil $(9.8 \%)^{25}$, and was similar to the rate found in the United Kingdom $(5 \%)^{7}$.

Phylogenetic analysis showed a wide variety of HAdVs. In this context, species A was found to be predominant (28.8\%), and HAdV type 12 was detected at the highest rate (17.3\%). Recently, HAdV type 12 was reported as the main cause of an AGE outbreak in Rio de Janeiro (83.3\%) involving children under 25 months of age in a low-income setting ${ }^{26}$. Although this genotype uses the gut as its main replication site, this was the first report in Brazil to relate such genotype to a specific outbreak. In the present study, five $(9.6 \%)$ out of 52 positive samples were genotyped as HAdV 31. This rate was lower than that reported in Beijing, China, between 2011 and 2012, where HAdV 31 was responsible for $15.7 \%$ of the children hospitalized with acute $\mathrm{AGE}^{27}$. These findings provide evidence that both HAdV type 12 and type 31 may cause sporadic cases or outbreaks.

Human adenovirus belonging to species $\mathrm{F}$ also accounted for a significant (26.9\%) proportion of diarrheal cases in our study. 
The prevalence of HAdV species F in our study was lower than that $(42.4 \%)$ reported through a survey conducted in Albania among hospitalized children, possibly because this type was related more intimately with severe $\mathrm{AGE}^{23}$. In line with our findings, a number of other studies have indicated that HAdV species $\mathrm{F}$ accounted for $4.4-9.8 \%$ of gastroenteritis cases among hospitalized children under three years of age ${ }^{26,28}$. In Belém, a 2003 surveillance study at both hospital and ambulatory level showed the presence of HAdV in $6.3 \%(24 / 380)$ of tested samples, with species F representing $58.3 \%$ of the HAdVpositive children $(14 / 24)^{9}$. It should be noted that different and somewhat less sensitive methods were used in these studies (including EIA). Moreover, some previous studies effectively addressed the presence of only enteric adenoviruses 40 and 41 , whereas the broad-range assay used in the present study can detect all known HAdV genotypes.

Other non-enteric HAdVs, such as species B, C, and D that are known to cause respiratory and ocular disease ${ }^{23,29,30}$, were also detected in our study. This observation may be explained by the ability all species have of replicating in the intestine and being excreted in the feces ${ }^{26,31}$. It is important to mention that all children who participated in this study presented diarrhea at the time of feces collection. While there are reports in the literature associating this clinical manifestation with respiratory disease ${ }^{26}$, in the present study the lack of clinical records of these children did not allow us to verify a correlation with respiratory infection. This could be considered a limitation of the present study.

We were able to detect four different $\mathrm{SaV}$ genotypes in our study, namely GI.1, GI.7, GII.1, and GV.2. A previous study in Belém showed that $\mathrm{SaV}$ was detected in $4.9 \%(15 / 305)$ of stool samples collected in 2003, and included genotypes GI.1 $(20 \%, 3 / 15)$, GI.2 (13.3\%, 2/15), and GII.1 $(66.7 \%, 10 / 15)^{32}$. Our results indicate that such genotypes were already circulating in Belém as early as the 1990's.

$\mathrm{SaV}$ GI.1 is known to be the predominant genotype worldwide. In Europe, this genotype was identified as the cause of 11 outbreaks of gastroenteritis; in most of them, the patients were adults older than 60 years $^{33}$. In addition, genotype GII.1 presents a high prevalence compared to other $\mathrm{SaV}$ genotypes. For example, in 2003, its frequency was $66.7 \%$ among children with AGE in the State of Pará, Brazil ${ }^{32}$. In South Africa, during the period 2009-2013, this genotype was considered predominant among children hospitalized due to $\mathrm{AGE}^{34}$.

Of note, studies conducted in four provinces of South Africa, from 2009 to 2013, as well as in a periurban community in Peru, from 2007 to 2010 , reported the detection of GI.7 strains in stool samples from children with gastroenteritis, at positivity rates of $1.8 \%(4 / 221)$ and $1.7 \%(5 / 299)$, respectively $y^{34,35}$. The first description of genotype GI.7 was made by analyzing all capsid proteins using universal primers for specific genogroups detected in samples from three prefectures (Chiba, Ehime, and Ishikawa) in Japan between 1999 and $2005^{17}$. In the present study, this genotype was described in a diarrheic case, demonstrating its circulation in Belém, Brazil, since 1990.

The GV genome group, which includes the GV.2 genotype, was initially reported in fecal samples from children hospitalized for AGE in Thailand ${ }^{36}$. In addition, the GV.2 genotype was found to be circulating during an outbreak of food-borne gastroenteritis in Japan ${ }^{37}$. In Brazil, there were reports of a GV.1 genotype in a fecal sample collected during a surveillance study in Rio de Janeiro during 2012-2014 ${ }^{38}$.

To the best of our knowledge, this is the first time that such unusual $\mathrm{SaV}$ genotypes, such as GI.7 and GV.2, have been documented in Brazil. Comprehensive molecular surveillance of the viral strains may be used in future studies targeting vaccine design. Furthermore, our results demonstrate that these genotypes were already in circulation, reinforcing the importance of using more effective techniques to facilitate their detection and understand their spreading.

The highest prevalence of HAdV infection, involving all species, was detected among children aged 18-24 months $(57.1 \%)$, even though the number of samples tested in this specific group was small. A study among children in Turkey found a lower prevalence (18.8\%) in the same age group, whereas a study on 13.5-month old children in Albania found a greater prevalence ${ }^{23,39}$. In Tanzania, the highest prevalence of HAdV gastroenteritis was noted among children aged 7-12 months $(62.5 \%, 15 / 24)^{22}$.

In our study, the highest positivity rate of $\mathrm{SaV}$ infection was also seen in the 18-24 months age group (19\%), a rate similar to that reported in São Luís, Maranhão, but different from that reported in Japan among children older than 36 months ${ }^{24,40}$. The prevalence of acute gastroenteritis in children less than two years of age reveals that this range is more predisposed to hydroelectrolytic disorders and more severe clinical conditions due to immunological immaturity and propensity for secondary infections.

The prevalence of HAdV infections in Belém did not differ significantly throughout the 2-year surveillance period, as no marked seasons are seen in this part of Brazil. The year encompasses a typical rainy season from December to May, followed by a drier (though with some rainfall) period from June to November. In contrast, a study conducted on adults without diarrhea in Esteio city, Rio Grande do Sul State, a part of southern Brazil characterized by well-defined seasons, reported a higher prevalence of HAdV infection during the summer (43.8\%), as compared with the winter $(21.6 \%)^{41}$.

Owing to the low positivity rate obtained $(5.2 \%, 9 / 172)$ and short time of surveillance (only two years), it was difficult to establish a seasonality of $\mathrm{SaV}$ infections. It is possible that, having the samples been collected more than 25 years ago and manipulated at times thereafter, results may have been influenced by these events. Besides that, $\mathrm{SaV}$ is an RNA virus, which makes it more unstable than HAdV, a DNA virus, and can be degraded more easily. Further and broader studies are therefore needed in an attempt to better understand the epidemiological features of $\mathrm{HAdV}$ and $\mathrm{SaV}$ as causes of infantile gastroenteritis in our region.

In conclusion, this study showed: 1) Circulation of HAdV and $\mathrm{SaV}$ in Belém, with HAdV detected at a high prevalence (43\%) among children in the community; 2) A great diversity of HAdV species, highlighting the higher prevalence of A and 
F species; 3) The first description of genotypes GI.7 and GV.2 of $\mathrm{SaV}$ in Brazil, and their circulation since 1990; 4) Higher HAdV and $\mathrm{SaV}$ frequencies in children aged 18-24 months, in line with published data; and 5) HAdV presence in almost every month of the studied years, highlighting the high circulation of this agent.

In summary, our findings will contribute substantially to the information already available about the circulation and genetic diversity of these viruses, creating a more complete picture of their molecular-epidemiological distribution, particularly in the Northern Region of Brazil, as well as in relation to the two $\mathrm{SaV}$ genotypes detected for the first time in the country.

\section{Acknowledgements}

We are grateful to all the staff of Norovirus and others Enteric Viruses Laboratory (LNVE). Thanks to all the children who participated in the study and their mothers, as well as to all the employees that conducted this study between 1990 and 1992, and helped with specimen collection. We give credit to the Coordination of Improvement of Higher Education Personnel (CAPES) for giving an important scholarship to the authors Larissa Costa and Thayara Portal.

\section{Financial support}

This research was supported by the Evandro Chagas Institute and Fundação Amazônia de Amparo a Estudos e Pesquisa do Pará [(FAPESPA), Edital: 006/2014 - Programa de auxílio à pesquisa/Projetos temáticos da FAPESPA: Saúde].

\section{Conflict of interest}

The authors declare that there is no conflict of interest

\section{REFERENCES}

1. Walker CL, Rudan I, Liu L, Nair H, Theodoratou E, Bhutta ZA, et al. Global burden of childhood pneumonia and diarrhoea. Lancet. 2013;38(9875):1405-16.

2. Hage E, Gerd Liebert U, Bergs S, Ganzenmueller T, Heim A. Human mastadenovirus type 70: a novel, multiple recombinant species D mastadenovirus isolated from diarrhoeal faeces of a haematopoietic stem cell transplantation recipient. J Gen Virol. 2015;96(9):2734-42.

3. Santos NOS, Romanos MTV, Wigg, MD. Introdução à virologia humana. 3th ed. Rio de Janeiro: Guanabara Koogan S.A. 2015; p. $190-224$.

4. Imade PE, Eghafona N. Viral Agents of Diarrhea in Young Children in Two Primary Health Centers in Edo State, Nigeria. Int J Microbiol. 2015(2015);Article ID 685821:1-5.

5. Elhag WI, Saeed HA, Omer el FE, Ali AS. Prevalence of rotavirus and adenovirus associated with diarrhea among displaced communities in Khartoum, Sudan. BMC Infect Dis. 2013;3:209.

6. Sdiri-loulizi K, Hassine M, Gharbi-Khelifi H, Aouni Z, Chouchane $\mathrm{S}$, Sakly N, et al. Molecular detection of genogroup I sapovirus in Tunisian children suffering from acute gastroenteritis. Virus Genes. 2011;43(1):6-12.

7. Cunliffe NA, Booth JA, Elliot C, Lowe SJ, Kitchin WSN, Nakagomi ONT, et al. Healthcare-associated Viral Gastroenteritis among Children in a Large Pediatric Hospital, United Kingdom. Emerg Infect Dis. 2010;16(1):55-62.
8. Andreasi MSA, Cardoso DDP, Fernandes SM, Tozetti IA, Borges AMT; Fiaccadori FS, et al. Adenovirus, Calicivirus and Astrovirus detection in fecal samples of hospitalized children with acute gastroenteritis from Campo Grande, MS, Brazil. Mem Inst Oswaldo Cruz. 2008;103(7):741-4.

9. Muller ECA, Morais MAA, Gabbay YB, Linhares AC. Ocorrência de adenovirus em crianças com gastrenterite aguda grave na cidade de Belém, Pará, Brasil. Rev Pan-Amaz Saude. 2010;1(3):49-55.

10. Ferreira CEO, Raboni SM, Pereira LA, Nogueira MB, Vidal LRR, Almeida SM. Viral acute gastroenteritis: clinical and epidemiological features of co-infected patients. Braz J Infect Dis. 2012;16 (3):267-72.

11. Amaral MS, Estevam GK, Penatti M, Lafontaine R, Lima IC, Spada $\mathrm{PK}$, et al. The prevalence of norovirus, astrovirus and adenovirus infections among hospitalised children with acute gastroenteritis in Porto Velho, state of Rondônia, western Brazilian Amazon. Mem. Inst. Oswaldo Cruz. 2015;110(2):215-21.

12. Mascarenhas JD, Leite JP, Gabbay YB, Freitas RB, Oliveira CS, Monteiro TA, et al. Rotavirus G serotypes and p[],G genotypes identified in cases of reinfection among children participating in a trial with rhesus-human reassortant tetravalent vaccine (RRV-TV) in Belém, Brazil. J Trop Pediatr. 2002;48(2):93-7.

13. Linhares AC, Gabbay YB, Mascarenhas JD, de Freitas RB, Oliveira $\mathrm{CS}$, Bellesi $\mathrm{N}$, et al. Immunogenicity, safety and efficacy of tetravalent rhesus-human, reassortant rotavirus vaccine in Belém, Brazil. Bull World Health Organ. 1996;74(5):491-500.

14. Gabbay YB, Leite JP, Oliveira DS, Nakamura LS, Nunes MR, Mascarenhas JD, et al. Molecular epidemiology of astrovirus type 1 in Belém, Brazil, as an agent of infantile gastroenteritis, over a period of 1810 years (1982-2000): Identification of two possible new lineages. Virus Res. 2007;129(2):166-74.

15. Allard A, Albinsson B, Wadell G. Rapid typing of human adenoviruses by a general PCR combined with restriction endonuclease analysis. J Clin Microbiol. 2001;39(2):498-505.

16. Jiang X, Huang PW, Zhong WM, Farkas T, Cubitt DW, Matson DO. Design and evaluation of a primer pair that detects both Norwalklike and Sapporo-like caliciviruses by RT-PCR. J Virol Methods. 1999;83:145-54.

17. Okada M, Yamashita Y, Oseto M, Shinozaki K. The detection of human sapoviruses with universal and genogroup-specific primers. Arch Virol, 2006;151:2503-9.

18. Oka T, Katayama K, Hansman GS, Kageyama T, Ogawa S, Wu F, etal. Detection of human sapovirus by real-time reverse transcriptionpolymerase chain reaction. J Med Virol. 2006;78(10):1347-53.

19. Kearse M, Moir R, Wilson A, Stones-Havas S, Cheung M, Sturrock $\mathrm{S}$, et al. Geneious basic: an integrated and extendable desktop software platform for the organization and analysis of sequence data. Bioinformatics. 2012;28(12):1647-9.

20. Minh BQ, Nguyen MAT, Haeseler AV. Ultrafast approximation for phylogenetic bootstrap. Mol Biol Evol. 2013;30(5):1188-95.

21. Ayres M, Ayres Jr M, Ayres DL, Santos AAS. BioEstat 5.0Aplicações Estatísticas nas Áreas das Ciências Bio-Médicas. 5th ed. Belém: Sociedade Civil Mamirauá CNPq; 2007. 290p.

22. Moyo SJ, Hanevik K, Blomberg B, Kommedal O, Nordbø SA, Maselle S, et al. Prevalence and molecular characterisation of human adenovirus in diarrhoeic children in Tanzania; a case control study. BMC InfectDis. 2014;14:666.

23. La Rosa G, Della Libera S, Petricca S, Iaconelli M, Donia D, Saccucci P, et al. Genetic Diversity of Human Adenovirus in Children with Acute Gastroenteritis, Albania, 2013-2015. Biomed Res Int. 2015 (2015);Article ID 142912: 1-7. 
24. Portal TM, Siqueira JA, Costa LC, Lima IC, Lucena MS, Bandeira RS, et al. Caliciviruses in hospitalized children, São Luís, Maranhão, 1997-1999: detection of norovirus GII.12. Braz J Microbiol. 2016;47(3):724-30.

25. Portes SAR, VolotãoEM, RochaMS, Rebelo MC, XavierMPTP, Assis RM, et al. A non-entericadenovirus A12 gastroenteritisoutbreak in Rio de Janeiro, Brazil. Mem Inst Oswaldo Cruz. 2016;111(6):403-6.

26. Liu L, Qian Y, Zhang Y, Deng J, Jia L, Dong H. Adenoviruses Associated with Acute Diarrhea in Children in Beijing, China. Plos One. 2014;9(2):1-6.

27. Linhares AC, Lanata CF, Hausdorff WP, Gabbay YB, Black RE. Reappraisal of the Peruvian and Brazilian titer tetravalent rhesushuman reassortant Rotavirus vaccine efficacy trial: analysis by severity of diarrhoea. Pediatr Infect Dis J. 1999;18(11):1001-6.

28. Li L, Phan TG, Nguyen TA, Kim KS, Seo JK, Shimizu H, et al. molecular epidemiology of adenovirus infection among pediatric population with diarrhea in Asia. Microbiol. Immunol. 2005;49(2):121-8.

29. Maranhão AG, Soares CC, Albuquerque MC, Santos N. Molecular epidemiology of adenovirus conjunctivitis in Rio de Janeiro, Brazil, between 2004 and 2007. Rev Inst Med Trop. 2009;51(4):227-9.

30. Pauly M, Hoppe E, Mugisha L, Petrzelkova K, Akoua-Koffi C, Couacy-Hymann E, et al. High prevalence and diversity of species D adenoviruses (HAdV-D) in human populations of four SubSaharan countries. Virol J. 2014;11:25.

31. Jiang SC. Human adenoviruses in water: Ocurrence and health implications: A critical review. Environ Sci Technol. 2006; 40(23):7132-40.

32. Aragão GC, Oliveira DS, Santos MC, Mascarenhas JDP, Oliveira CS, Linhares AC, et al. Molecular characterization of norovirus, sapovirus and astrovirus in children with acute gastroenteritis from Belém, Pará, Brazil. Rev Pan-Amaz Saude. 2010;1(1):149-58.
33. Svraka S, Vennema H, Van der veer B, Hedlund KO, Thorhagen M, Siebenga $\mathrm{J}$, et al. Epidemiology and genotype analysis of emerging sapovirus-associated infections across Europe. J Clin Microbiol. 2010;48(6):2191-8

34. Murray TY, Nadan S, Page NA, Taylor MB. Diverse sapovirus genotypes identified in children hospitalised with gastroenteritis in selected regions of South Africa. J Clin Virol. 2016;76:24-9.

35. Liu X, Jahuira H, Gilman RH, Alva A, Cabrera L, Okamoto M, et al. Etiological role and repeated infections of sapovirus among children aged less than 2 years in a cohort study in a peri-urban community of Peru. J Clin Microbiol. 2016;54(6):1598-604.

36. Guntapong R, Hansman GS, Oka T, Ogawa S, Kageyama T, Pongsuwanna $\mathrm{Y}$, et al. Norovirus and sapovirus infections in Thailand. Jpn J Infect Dis. 2004;57(6):276-8.

37. Shibata S, Sekizuka T, Kodaira A, Kuroda M, Haga K, Doan YH, et al. Complete Genome Sequence of a Novel GV.2 Sapovirus Strain, NGY-1, Detected from a Suspected Foodborne Gastroenteritis Outbreak. Genome Announc. 2015;3(1):e01553-14

38. Fioretti JM, Rocha MS, Fumian TM, Ginuino A, da Silva TP, de Assis MR, et al. Occurrence of human sapoviruses in wastewater and stool samples in Rio De Janeiro, Brazil. J Appl Microbiol. 2016;121(3):855-62.

39. Biçer S, Sahin GT, Koncay B, Gemici H, Siraneci R, Öztürk NY, et al. Incidence assessment of rotavirus and adenovirus associated acute gastroenteritis cases in early childhood. Infez Med. 2011;19(2):113-9.

40. Phan TG, Okitsu S, Muller WE, Kohno H, Ushijima H. Novel recombinant sapovirus, Japan. Emerg Infect Dis. 2006;12:865-7.

41. Vetter MR, Staggemeier R, Vecchia AD, Henzel A, Rigotto C, Spilki FR. Seasonal variation on the presence of adenoviruses in stools from non-diarrheic patients Braz J Microbiol. 2015;46(1): $749-52$. 